\title{
Autoresonant switching of the magnetization in single-domain nanoparticles: Two-level theory
}

\author{
Guillaume Klughertz \\ Institut de Physique et Chimie des Matériaux de Strasbourg, CNRS and Université de Strasbourg, \\ Boîte Postale 43, F-67034 Strasbourg, France \\ Lazar Friedland \\ Racah Institute of Physics, Hebrew University of Jerusalem, Jerusalem 91904, Israel \\ Paul-Antoine Hervieux and Giovanni Manfredi* \\ Institut de Physique et Chimie des Matériaux de Strasbourg, CNRS and Université de Strasbourg, \\ Boîte Postale 43, F-67034 Strasbourg, France
}

(Received 20 October 2014; revised manuscript received 16 March 2015; published 30 March 2015)

\begin{abstract}
The magnetic moment of a single-domain nanoparticle can be effectively switched on an ultrashort time scale by means of oscillating (microwave) magnetic fields. This switching technique can be further improved by using fields with time-dependent frequency (autoresonance). Here, we provide a full theoretical framework for the autoresonant switching technique, by exploiting the analogy between the magnetization state of an isolated nanoparticle and a two-level quantum system, whereby the switching process can be interpreted as a population transfer. We derive analytical expressions for the threshold amplitude of the microwave field, with and without damping, and consider the effect of thermal fluctuations. Comparisons with numerical simulations show excellent agreement.
\end{abstract}

DOI: 10.1103/PhysRevB.91.104433

PACS number(s): 75.78. -n, 75.78.Jp, 75.20.-g, 05.45.-a

\section{INTRODUCTION}

Since the pioneering work of Frenkel and Dorfman [1], the unusual properties of single-domain magnetic nanoparticles have stimulated a great many theoretical and experimental investigations. The theoretical understanding of their behavior is of paramount importance for future applications in a wide variety of areas, ranging from magnetic data storing to waste water decontamination [2] and medical diagnostics and treatments [3-5]. In particular, the ability to precisely control the magnetization dynamics constitutes a major advantage, or even, in some cases, an essential requirement [6-10].

In the absence of any external perturbations (magnetic field, temperature), the macrospin of a monodomain nanoparticle with uniaxial anisotropy can only point along two antiparallel orientations along the easy axis of magnetization. The energy barrier separating these two orientations being proportional to the volume of the nanoparticle, it can easily be overcome either by applying an external magnetic field or because of thermal fluctuations. When the macrospin switches spontaneously and repeatedly between the two orientations under the action of the temperature, the magnetic moment is said to be in the superparamagnetic regime. This random behavior is obviously undesirable for a reliable control of the magnetization dynamics. Increasing the magnetic anisotropy of the nanoparticle solves this issue [11] (because it raises the energy barrier), but in turn also increases the switching field required to reverse the magnetization to values that are barely workable in experimental setups. An astute workaround [12,13], relying on the combined effect of a static and an oscillating magnetic field (in the microwave domain), can significantly reduce the amplitude of the switching field [14]. This technique is more efficient

\footnotetext{
*manfredi@unistra.fr
}

when the microwave field frequency is time dependent and synchronized with the precession frequency of the magnetic moment. Such synchronization requires a fine-tuned feedback mechanism in order to stay in resonance with the magnetic moment [15]. However, the use of such a feedback mechanism can be costly and cumbersome in practical situations. Our recent work showed that this limitation can be overcome using the concept of autoresonance [16].

The autoresonance mechanism [17] consists in driving to high amplitudes a nonlinear oscillator (in the present case, the magnetic moment) using an external excitation with time-dependent frequency (here, a rotating magnetic field). When the driving amplitude is above a certain threshold and its frequency passes through the linear resonant frequency of the system, the oscillator will be unswervingly phaselocked to the excitation and will therefore be driven to very high amplitudes. This phenomenon has been observed in atomic systems [18,19], plasmas [20,21], fluids [22], and semiconductor quantum wells [23]. Some earlier numerical and analytical studies [24-26] pointed out the efficiency of a chirped microwave field to induce the reversal of the magnetic moment in a nanoparticle, but did not exploit the analytical tools provided by the autoresonance theory.

In a recent work [16], we showed that autoresonance works very efficiently for the control of the magnetization reversal in magnetic nanoparticles. With this technique, it was possible to reduce by around 30\% the strength of the applied static field compared to other switching methods. In addition, the autoresonant excitation does not require any fine-tuned feedback mechanism. Our previous results [16] were supported essentially by numerical simulations of the Landau-Lifschitz-Gilbert (LLG) equation. Here, we provide a sound theoretical framework for these results, using a mathematical analogy between the LLG equation and a two-level system first developed by Feynman [27]. We will 
first examine the simpler case of an undamped system (precessing magnetic moment), before analyzing the effect of damping and thermal fluctuations. The theoretical results will be compared to numerical simulations of the full LLG equation.

\section{MODEL}

The physical system we consider is a single-domain magnetic nanoparticle, with uniaxial anisotropy directed along $\widehat{e}_{z}$. We assume that such a particle is well described, in the macrospin approximation, by a magnetic moment $\mathbf{M}$, with $|\mathbf{M}|=\mu_{s}$. In this case, the magnetization dynamics is governed by the LLG equation

$$
\frac{d \mathbf{M}}{d t}=-\gamma \mathbf{M} \times \mathbf{B}-\frac{\lambda \gamma}{\mu_{s}} \mathbf{M} \times(\mathbf{M} \times \mathbf{B}),
$$

where the effective magnetic field $\mathbf{B}$ consists of an anisotropy field, an external static field $\mathbf{B}_{\mathrm{dc}}=-B_{0} \widehat{e}_{z}$, and a circularly polarized (driving) component of amplitude $b$ rotating in the $(x, y)$ plane [16]:

$$
\mathbf{B}=\left(\frac{2 K V}{\mu_{s}^{2}} M_{z}-B_{0}\right) \widehat{e}_{z}+b\left(\cos \varphi_{d} \widehat{e}_{x}+\sin \varphi_{d} \widehat{e}_{y}\right) .
$$

Here, $\varphi_{d}=2 \pi\left(f_{r} t-\alpha t^{2} / 2\right)$, so that the driving frequency $\omega_{d}=2 \pi\left(f_{r}-\alpha t\right)$ is chirped linearly in time. The geometry of this configuration is shown in Fig. 1.

Typical parameters [28] for 3-nm-diameter cobalt nanoparticles are a gyromagnetic ratio $\gamma=1.76 \times 10^{11}(\mathrm{Ts})^{-1}$, an anisotropy constant $K=2.2 \times 10^{5} \mathrm{~J} / \mathrm{m}^{3}$, a volume $V=$ $14.1 \times 10^{-27} \mathrm{~m}^{3}$, and a magnetization at saturation $\mu_{s}=$ $2.36 \times 10^{-20} \mathrm{~J} / \mathrm{T}$.

It is convenient to use dimensionless variables and parameters, defined as

$$
\widetilde{\mathbf{M}}=\frac{\mathbf{M}}{\mu_{s}}, \widetilde{\mathbf{B}}=\frac{\gamma \mathbf{B}}{(2 \pi \alpha)^{1 / 2}}, \tau=(2 \pi \alpha)^{1 / 2} t .
$$

Equation (1) can thus be rewritten as

$$
\frac{d \tilde{\mathbf{M}}}{d \tau}=\widetilde{\mathbf{B}} \times \tilde{\mathbf{M}}-\lambda(\widetilde{\mathbf{B}} \times \tilde{\mathbf{M}}) \times \tilde{\mathbf{M}}
$$

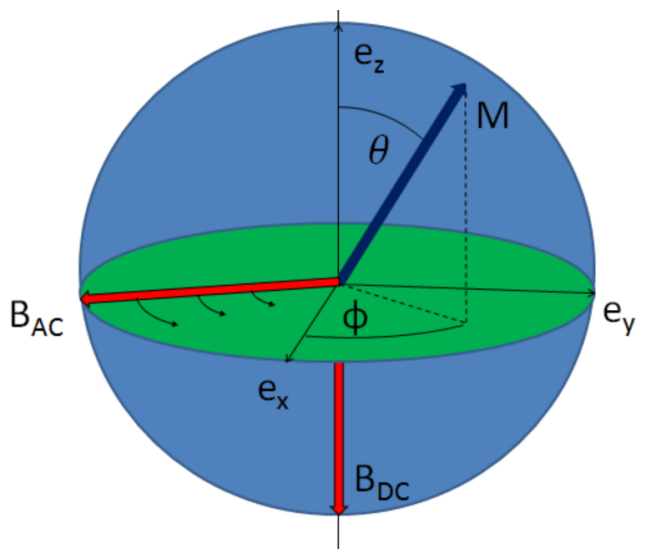

FIG. 1. (Color online) Geometric configuration of the nanoparticle with its magnetic moment $\mathbf{M}$, the static field $\mathbf{B}_{\mathrm{dc}}$, and the time-dependent ac field $\mathbf{B}_{\mathrm{ac}}(t)$. where

$$
\begin{gathered}
\widetilde{\mathbf{B}}=\left(a_{1} \tilde{M}_{z}-a_{2}\right) \widehat{e}_{z}+\varepsilon\left(\cos \varphi_{d} \widehat{e}_{x}+\sin \varphi_{d} \widehat{e}_{y}\right), \\
a_{1}=\frac{2 \gamma K V}{\mu_{s}(2 \pi \alpha)^{1 / 2}}, a_{2}=\frac{\gamma B_{0}}{(2 \pi \alpha)^{1 / 2}}, \varepsilon=\frac{\gamma b}{(2 \pi \alpha)^{1 / 2}},
\end{gathered}
$$

and $\varphi_{d}=\omega_{0} \tau-\tau^{2} / 2$, with $\omega_{0}=2 \pi f_{r}(2 \pi \alpha)^{-1 / 2}$. Initially, the magnetic moment is oriented along the $z$ direction, i.e., $\widetilde{\mathbf{M}}(0)=\widehat{e}_{z}$.

We showed recently [16] that, using the chirped excitation defined in Eq. (2), one can very efficiently and rapidly reverse the magnetization. In the next sections, we will provide a theoretical explanation for those numerical results.

\section{ANALOGY WITH A TWO-LEVEL SYSTEM}

We first consider the dissipationless problem $(\lambda=0)$ :

$$
\frac{d \tilde{\mathbf{M}}}{d \tau}=\widetilde{\mathbf{B}} \times \widetilde{\mathbf{M}}
$$

We adopt Feynman's equivalence [27] between Eq. (7) and the following "quantum" two-level system for the complex quantities $A_{1}$ and $A_{2}$ :

$$
\begin{gathered}
i \frac{d A_{1}}{d \tau}=\frac{\kappa_{0}}{2} A_{1}+\kappa A_{2}, \\
i \frac{d A_{2}}{d \tau}=-\frac{\kappa_{0}}{2} A_{2}+\kappa^{*} A_{1},
\end{gathered}
$$

where $\widetilde{\mathbf{M}}$ is related to $A_{1,2}$ as follows:

$$
\begin{aligned}
\tilde{M}_{x} & =A_{1} A_{2}^{*}+A_{1}^{*} A_{2}, \\
\tilde{M}_{y} & =i\left(A_{1} A_{2}^{*}-A_{1}^{*} A_{2}\right), \\
\tilde{M}_{z} & =\left|A_{1}\right|^{2}-\left|A_{2}\right|^{2},
\end{aligned}
$$

and

$$
\begin{aligned}
& \widetilde{B}_{x}=\kappa+\kappa^{*}, \\
& \widetilde{B}_{y}=i\left(\kappa-\kappa^{*}\right), \\
& \widetilde{B}_{z}=\kappa_{0} .
\end{aligned}
$$

Note that Eq. (8) conserves the total "population" of the two levels (normalized to unity), i.e., $\left|A_{1}\right|^{2}+\left|A_{2}\right|^{2}=1$. By definition, $\kappa=\frac{1}{2}\left(\widetilde{B}_{x}-i \widetilde{B}_{y}\right)=\frac{\varepsilon}{2} \exp \left(-i \varphi_{d}\right)$ and $\kappa_{0}=a_{1}\left(\left|A_{1}\right|^{2}-\right.$ $\left.\left|A_{2}\right|^{2}\right)-a_{2}=a_{1}-a_{2}-2 a_{1}\left|A_{2}\right|^{2}$, so that Eqs. (8) and (9) become

$$
\begin{aligned}
& i \frac{d A_{1}}{d \tau}=\frac{1}{2}\left(\omega_{0}-2 a_{1}\left|A_{2}\right|^{2}\right) A_{1}+\frac{\varepsilon}{2} e^{-i \varphi_{d}} A_{2}, \\
& i \frac{d A_{2}}{d \tau}=-\frac{1}{2}\left(\omega_{0}-2 a_{1}\left|A_{2}\right|^{2}\right) A_{2}+\frac{\varepsilon}{2} e^{+i \varphi_{d}} A_{1},
\end{aligned}
$$

where $\omega_{0}=a_{1}-a_{2}$ is the linear frequency in the problem and we solve Eqs. (12) and (13) subject to the initial conditions $A_{1}(0)=1$ and $A_{2}(0)=0$.

Autoresonance in this system of coupled nonlinear Schrödinger (NLS) equations was first studied for mode conversion problems in plasmas [29] and later for a nonlinear optics application [30]. Thus, many characteristic features of the autoresonant evolution of magnetization can now be 
studied using the results of these earlier works. Here, we focus on the autoresonance threshold phenomenon in the process of capture into resonance in the small-nonlinearity limit of the driven, chirped frequency problem. To this end, we define new complex amplitudes,

$$
\begin{aligned}
& \bar{A}_{1}=A_{1} \exp \left(\frac{i}{2} \int \kappa_{0}(\tau) d \tau\right), \\
& \bar{A}_{2}=A_{2} \exp \left[-i\left(\varphi_{d}-\frac{1}{2} \int \kappa_{0}(\tau) d \tau\right)\right],
\end{aligned}
$$

for which Eqs. (12) and (13) become

$$
\begin{gathered}
i \frac{d \bar{A}_{1}}{d \tau}=\frac{\varepsilon}{2} \bar{A}_{2}, \\
i \frac{d \bar{A}_{2}}{d \tau}+\left(\tau-2 a_{1}\left|\bar{A}_{2}\right|^{2}\right) \bar{A}_{2}=\frac{\varepsilon}{2} \bar{A}_{1} .
\end{gathered}
$$

We also assume that the passage through the linear resonance occurs at $\tau=0$, i.e., $\omega_{d}=\omega_{0}-\tau$. In the weakly nonlinear excitation regime, for sufficiently small $\varepsilon$ and $\bar{A}_{2}$, the conservation equation yields $\bar{A}_{1} \approx 1$ and Eq. (14) guarantees that $\bar{A}_{1}$ remains initially constant. Then, Eq. (15) can be written as

$$
i \frac{d \psi}{d \tau}+\left(\tau-|\psi|^{2}\right) \psi=\mu,
$$

where $\psi=\left(2 a_{1}\right)^{1 / 2} \bar{A}_{2}$ and $\mu=\varepsilon\left(a_{1} / 2\right)^{1 / 2}$. This is the usual NLS-type equation describing the autoresonance threshold phenomenon [17]. For the initial condition $\psi=0$ (at $t=$ $-\infty)$, this equation predicts bifurcation from a bounded solution at $t=+\infty$ to the autoresonantly ever-increasing solution $|\psi| \sim \tau^{1 / 2}$ as the driving parameter $\mu$ exceeds a certain threshold $\mu_{\text {th }}$. In undamped systems, earlier numerical calculations [31,32] yielded the value $\mu_{\text {th }}=0.41$, so that

$$
b_{\mathrm{th}}=\frac{0.41(2 \pi \alpha)^{3 / 4}}{\gamma^{3 / 2}\left(K V / \mu_{s}\right)^{1 / 2}} .
$$

Using our physical parameters we get $b_{\text {th }}=6.08 \times 10^{-17} \alpha^{3 / 4}$. Numerical simulations of Eq. (7) (see Fig. 2) show that $b_{\text {th }}$ is

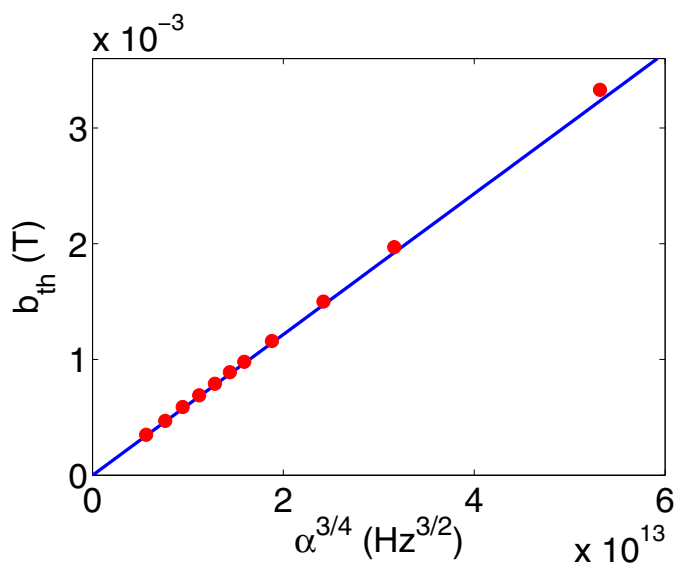

FIG. 2. (Color online) Threshold amplitude as a function of the chirp rate $\alpha^{3 / 4}$. The red dots are numerical results obtained by solving the full LLG equation. The straight line is the theoretical result from Eq. (17). indeed proportional to $\alpha^{3 / 4}$, with the correct proportionality constant as given by the theoretical expression (17).

Note that if one uses a linearly polarized driving magnetic field, the same analysis predicts twice as large a threshold $b_{\text {th }}$, in accordance with earlier numerical results [16].

\section{TRANSITION FROM AUTORESONANCE TO LINEAR LANDAU-ZENER PROBLEM}

The following analysis is somewhat similar to that developed for the quantum versus classical phase-locking transition in a frequency-chirped nonlinear oscillator [33]. We observe that Eqs. (14) and (15) possess two dimensionless parameters: the nonlinearity parameter $a_{1}$ and the driving parameter $\varepsilon$, which can be given another physical interpretation. Indeed, there exist three characteristic time scales in our original magnetization problem, i.e., the characteristic driving frequency sweep time $T_{S}=1 / \sqrt{2 \pi \alpha}$, the nonlinearity time scale $T_{N L}=2 \gamma K V / \mu_{s}$, and the characteristic time associated with the driving amplitude (inverse Rabi frequency in the two-level system) $T_{R}=(\gamma b)^{-1}$. The ratios of these three characteristic times yield the two dimensionless parameters in our nonlinear two-level problem:

$$
a_{1}=T_{S} / T_{N L} \text { and } \varepsilon=T_{S} / T_{R} .
$$

Here, we shall discuss the question of efficient transfer of population between the two levels (corresponding to efficient magnetization switching) as the result of passage through the linear resonance in our two-parameter space $\left(\varepsilon, a_{1}\right)$. Because of the extended parameter space, one can expect that this problem will differ from that of the singleparameter approximation of Eq. (16). Figure 3 shows values of $\left|\bar{A}_{2}\right|^{2}$ at large positive $\tau$, obtained from numerical solutions of Eqs. (14) and (15) with the initial condition $\bar{A}_{2}=0$ at large negative $\tau$. In the figure, we use the axis $1 / \sqrt{a_{1}}$ and $\varepsilon$, since, as shown above, the autoresonance threshold scales as $\varepsilon_{\mathrm{th}}^{A R}=0.59 / \sqrt{a_{1}}$. This linear dependence

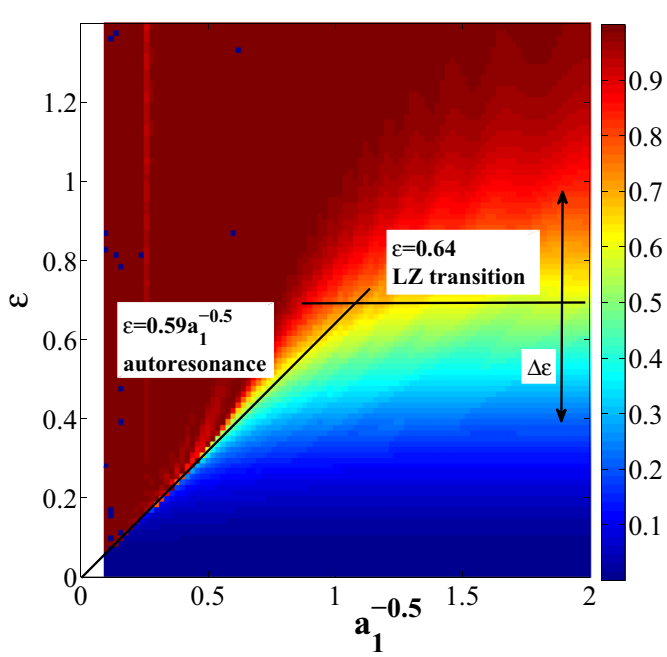

FIG. 3. (Color online) Amplitude of the $\left|A_{2}\right|^{2}$ level in the twoparameter space $\left(\epsilon, a_{1}^{-0.5}\right)$, obtained from numerical solutions of Eqs. (14) and (15). Regions where $\left|A_{2}\right|^{2}$ is larger are those of efficient population transfer (i.e., efficient magnetization switching). 
on $1 / \sqrt{a_{1}}$ of the sharp transition to autoresonance can be clearly seen for sufficiently large values of $a_{1}$, say $a_{1}^{-0.5}<0.4$ (bottom left part of the figure). For a weaker nonlinearity (smaller values of $a_{1}$, on the right in the figure), the process gradually becomes that of the linear Landau-Zener (LZ) transition [34]. The latter is characterized by the following dependence on the driving parameter: $\left|\bar{A}_{2}\right|^{2}=\exp \left(-\pi \varepsilon^{2} / 2\right)$.

This transition has a characteristic width $\Delta \varepsilon=1 / \sqrt{\pi} \approx$ 0.56 . The value of $\varepsilon$ for which $\left|\bar{A}_{2}\right|^{2}=0.5$ can be used as the threshold for efficient excitation (magnetization switching) in this LZ limit, i.e., $\varepsilon_{\mathrm{th}}^{\mathrm{LZ}} \approx 0.64$. All these predictions are in full agreement with the results of the simulations shown in Fig. 3.

\section{EFFECT OF DAMPING AND THERMAL FLUCTUATIONS}

We now include the dissipative $\lambda$ term in Eq. (4). We write this equation as

$$
\frac{d \tilde{\mathbf{M}}}{d \tau}=\widetilde{\mathbf{B}}^{\prime} \times \tilde{\mathbf{M}},
$$

where $\widetilde{\mathbf{B}}^{\prime}=\widetilde{\mathbf{B}}-\lambda \widetilde{\mathbf{B}} \times \widetilde{\mathbf{M}}$. Next, we again apply Feynman's formalism, i.e., we associate this problem to a two-level system, Eqs. (8) and (9), where now, after some algebra (see Appendix A) one obtains the analog of Eq. (16) with the damping term

$$
i \frac{d \psi}{d \tau}+\left(\tau-|\psi|^{2}+i \nu / 2\right) \psi=\mu,
$$

where $v=2 \lambda \omega_{0}$. This two-parameter equation is characteristic of other autoresonant problems in the presence of damping (see the theory [35] and related experiments [31]). It was shown earlier [31] that the threshold for transition to autoresonance is modified by the damping and becomes

$$
\mu_{\mathrm{th}}=0.41\left(1+1.06 v+0.67 v^{2}\right)+O\left(v^{3}\right) .
$$

Numerical simulations (Fig. 4) show an excellent agreement with the above theoretical result.

We also note that, since $v$ goes as $\alpha^{-1 / 2}$, the scaling of the threshold for the driving field in the presence of dissipation

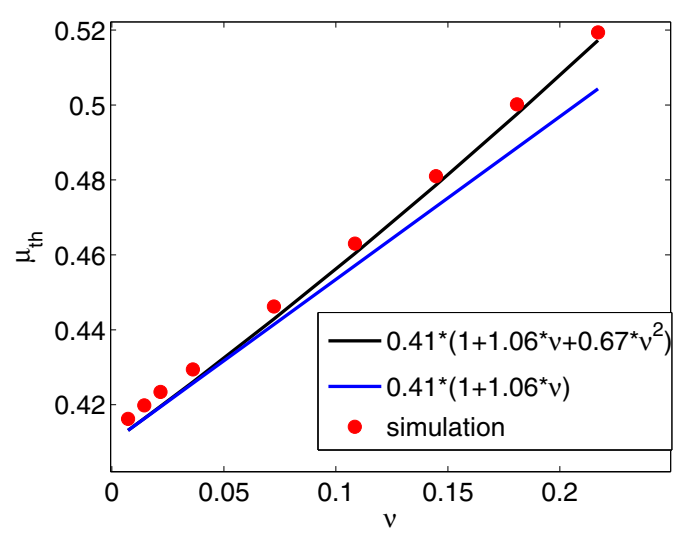

FIG. 4. (Color online) Rescaled threshold amplitude $\mu_{\text {th }}$ as a function of the damping $v$. Simulation data are represented by red dots; the solid lines correspond to the theoretical result to first (blue) and second (black) order in $v$, from Eq. (21). deviates from the law $b_{\text {th }} \sim \alpha^{3 / 4}$, which is typical of the dissipationless regime (Fig. 2).

So far, we have only considered the zero-temperature (deterministic) case. We now study the influence of thermal effects on the magnetization reversal. According to the NéelBrown theory [36,37], thermal effects can be taken into account by adding a fluctuating magnetic field $\delta \mathbf{B}$, with zero mean and autocorrelation function given by

$$
\left\langle\delta B_{i}(t) \delta B_{j}\left(t^{\prime}\right)\right\rangle=\eta \delta_{i j} \delta\left(t-t^{\prime}\right),
$$

where $\eta=\frac{2 k_{B} T \lambda}{\gamma \mu_{s}}$. Thus, the LLG equation (19) can be rewritten as

$$
\frac{d \tilde{\mathbf{M}}}{d t}=\gamma \mathbf{B}^{\prime} \times \tilde{\mathbf{M}},
$$

where $\mathbf{B}^{\prime}=\mathbf{B}-\lambda \mathbf{B} \times \tilde{\mathbf{M}}+\delta \mathbf{B}$, with $\mathbf{B}=B_{0}^{\prime} \widehat{\boldsymbol{e}}_{z}$ and $B_{0}^{\prime}=$ $2 K V / \mu_{s}-B_{0}$.

At finite temperature, the thermal fluctuations drive the magnetic moment away from the $z$ axis and bring it to a randomly distributed orientation before the autoresonant field is activated [38]. After the thermal equilibrium is established, the chirped drive is switched on. During the autoresonant excitation the thermal effects are present, but their effect is negligible compared to the driving field. Therefore, one can assume [39] that the thermal noise enters the problem only through the initial condition on the amplitude $A \equiv|\psi|$ and the phase of $\psi$ in Eq. (20). This randomness in the initial distribution creates a finite width in the transition to the autoresonant regime, so that the threshold is no longer sharp as in the zero-temperature case [16]. The thermal width of the threshold for the driving parameter $\mu$ can be written in the form [39]

$$
\Delta \mu=\sqrt{2 \pi} \kappa \sigma
$$

where $\kappa=0.245$ and $\sigma$ is the thermal width of the distribution $P\left(A_{0}\right)=\left(A_{0} / \sigma^{2}\right) \exp \left(-A_{0}^{2} / 2 \sigma^{2}\right)$ of the initial values $A_{0}=$ $A(0)$.

In order to compute $\sigma$, we again transform to the quantum two-level problem (see Appendix B) and find

$$
\sigma^{2}=\frac{k_{B} T a_{1}}{2 \mu_{s} B_{0}^{\prime}} .
$$

The substitution of this result into Eq. (24) gives the desired width for the autoresonant threshold parameter:

$$
\Delta \mu=\kappa \sqrt{\frac{\pi k_{B} T a_{1}}{\mu_{s} B_{0}^{\prime}}},
$$

or, expressed as the amplitude of the driving magnetic field,

$$
\Delta b_{\mathrm{th}}=\frac{2 \kappa \pi}{\gamma} \sqrt{\frac{k_{B} T \alpha}{\mu_{s} B_{0}^{\prime}}} .
$$

This analytical expression was checked against numerical results obtained by solving the full LLG equation. Following the procedure described in our earlier work [16], the transition width can be quantified by the capture probability $P\left(b_{\mathrm{ac}}\right)$, defined as the probability for a magnetic moment to switch under the action of an autoresonant field of amplitude $b_{\text {ac }}$. The transition width is mathematically defined as the inverse slope 


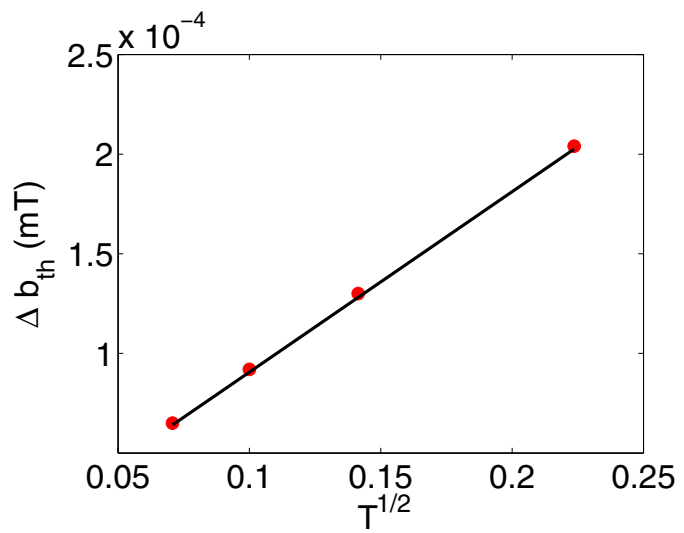

FIG. 5. (Color online) Width of the threshold for the driving field in the presence of thermal noise, as a function of the square root of the temperature. The red dots are numerical results obtained by solving the full LLG equation. The straight line is the theoretical result of Eq. (27).

of $P\left(b_{\mathrm{ac}}\right)$ computed at the inflexion point of the curve. Figure 5 shows the comparison between the numerically computed width and the theoretical expression, Eq. (27). The agreement is very good.

Note also that the phenomenological damping parameter $\lambda$ does not enter this formula. Furthermore, the width $\Delta b_{\text {th }}$ decreases for increasing $B_{0}^{\prime} \equiv 2 K V / \mu_{s}-B_{0}$. Thus, it appears that removing the static magnetic field $B_{0}$ can even be beneficial for the transition to the autoresonant regime by reducing the transition width.

\section{CONCLUSIONS}

In this paper, we developed a full theory of the autoresonant switching of the magnetization in single-domain nanoparticles, a technique that we had proposed in an earlier work [16]. The theory-which is based on the Feynman equivalence between the magnetization dynamics and a two-level quantumlike system-allowed us to predict quantitatively the autoresonant threshold, as well as the effect of damping and thermal noise on the efficiency of the switching. The theoretical estimates were in excellent agreement with the numerical simulations of the full LLG equation. This study corroborates our earlier finding that the magnetization of a nanoparticle can be reversed efficiently using a chirped microwave magnetic field - and this without any feedback control or other fine-tuning of the driving parameters.

The present work focused on the dynamics of isolated magnetic nanoparticles. For an assembly of densely packed nanoparticles-a common situation in experiments-dipolar interactions may play a significant role $[16,40]$. Depending on the spatial arrangement of the nanoparticles and the orientations of their anisotropy axes, the interactions may even favor the coherent switching of all the particles, as we showed recently using a mean field approach [16]. The present two-level model may shed more light on this type of coherent effect, which has many potential practical applications.

\section{ACKNOWLEDGMENTS}

We acknowledge the financial support of the Agence Nationale de la Recherche (France) through the projects Equipex UNION (Grant No. ANR-10-EQPX-52-01) and Labex NIE. L.F. acknowledges the support of the Israel Science Foundation (Israel).

\section{APPENDIX A: DAMPING}

As the addition of damping modifies the form of the effective field, we must recalculate $\kappa$ :

$$
\begin{aligned}
\kappa & =\frac{1}{2}\left(\widetilde{B}_{x}^{\prime}-i \widetilde{B}_{y}^{\prime}\right) \\
& =\frac{\varepsilon}{2} e^{-i \varphi_{d}}-i \frac{\lambda}{2}\left[\varepsilon \widetilde{M}_{z} e^{-i \varphi_{d}}-2\left(a_{1} \tilde{M}_{z}-a_{2}\right) A_{1} A_{2}^{*}\right]
\end{aligned}
$$

or, approximately,

$$
\kappa=\frac{\varepsilon}{2} e^{-i \Theta}+i \lambda\left(a_{1} \tilde{M}_{z}-a_{2}\right) A_{1} A_{2}^{*},
$$

where $\Theta=\varphi_{d}+\lambda \widetilde{M}_{z}$. Similarly, one finds

$\kappa_{0}=\widetilde{B}_{z}^{\prime}=a_{1} \widetilde{M}_{z}-a_{2}+\lambda \varepsilon\left(A_{1} A_{2}^{*} e^{-i \varphi_{d}}-c c\right) \approx a_{1} \widetilde{M}_{z}-a_{2}$,

and $c c$ stands for complex conjugate. Then the system in Eqs. (8) and(9) becomes

$$
\begin{aligned}
& i \frac{d A_{1}}{d \tau}=\frac{\kappa_{0}}{2} A_{1}+\frac{\varepsilon}{2} A_{2} e^{-i \Theta}+i \lambda\left(a_{1} \tilde{M}_{z}-a_{2}\right) A_{1}\left|A_{2}\right|^{2}, \\
& i \frac{d A_{2}}{d \tau}=-\frac{\kappa_{0}}{2} A_{2}+\frac{\varepsilon}{2} A_{1} e^{i \Theta}-i \lambda\left(a_{1} \tilde{M}_{z}-a_{2}\right)\left|A_{1}\right|^{2} A_{2} .
\end{aligned}
$$

We now proceed to the threshold calculation and use the weakly nonlinear limit $\left(\left|A_{2}\right|^{2} \ll 1,\left|A_{1}\right|^{2}=1-\left|A_{2}\right|^{2} \approx 1\right)$ of Eqs. (A2) and (A3) to obtain

$$
\begin{gathered}
i \frac{d A_{1}}{d \tau}=\frac{\kappa_{0}}{2} A_{1}, \\
i \frac{d A_{2}}{d \tau}=-\frac{\kappa_{0}}{2} A_{2}+\frac{\varepsilon}{2} e^{i \Theta} A_{1}-i \lambda \omega_{0} A_{2},
\end{gathered}
$$

where, as before, $\omega_{0}=a_{1}-a_{2}$ denotes the linear resonance frequency in the dissipationless problem. The first equation in this system yields $A_{1}=\exp \left(-i \kappa_{0} \tau / 2\right)$, so the second equation can be written as

$$
i \frac{d A_{2}}{d \tau}=-\frac{\kappa_{0}}{2} A_{2}+\frac{\varepsilon}{2} e^{i\left(\Theta-\kappa_{0} \tau / 2\right)}-i \lambda \omega_{0} A_{2} .
$$

Here, we introduce a new amplitude, $\bar{A}_{2}=A_{2} \exp [-i(\Theta-$ $\left.\kappa_{0} \tau / 2\right)$ ], to get

$$
i \frac{d \bar{A}_{2}}{d \tau}+\left(\omega_{0}-\omega_{d}-2 a_{1}\left|\bar{A}_{2}\right|^{2}+i \lambda \omega_{0}\right) \bar{A}_{2}=\frac{\varepsilon}{2},
$$

where the small term $\lambda d \widetilde{M}_{z} / d \tau$ on the left-hand side was neglected. Equation (A7) differs from Eq. (15) by an additional term, $i \lambda \omega_{0} \bar{A}_{2}$, only. Then, as before, by defining $\psi=$ $\left(2 a_{1}\right)^{1 / 2} \bar{A}_{2}$ and $\mu=\varepsilon\left(a_{1} / 2\right)^{1 / 2}$, the last equation becomes

$$
i \frac{d \psi}{d \tau}+\left(\tau-|\psi|^{2}+i \nu / 2\right) \psi=\mu,
$$

where $v=2 \lambda \omega_{0}$ 


\section{APPENDIX B: THRESHOLD WIDTH}

We rewrite Eq. (23) as a two-level system:

$$
\begin{gathered}
i \frac{d A_{1}}{d t}=\frac{\Omega_{0}}{2} A_{1}+\Omega A_{2}, \\
i \frac{d A_{2}}{d t}=-\frac{\Omega_{0}}{2} A_{2}+\Omega^{*} A_{1},
\end{gathered}
$$

where

$$
\begin{gathered}
\Omega_{0}=\gamma\left(B_{0}^{\prime}+\delta B_{z}\right), \\
\Omega=\frac{\gamma}{2}\left(\delta B_{x}-i \delta B_{y}\right)+i \lambda \Omega_{0} A_{1} A_{2}^{*} .
\end{gathered}
$$

We solve Eqs. (B1) and (B2) subject to the initial conditions $A_{1}(0)=1$ and $A_{2}(0)=0$. Then, for small excitations $\left(\left|A_{2}\right|<<1\right)$, one can approximate Eq. (B1) by

$$
i \frac{d A_{1}}{d t} \approx \frac{\Omega_{0}}{2} A_{1}, \quad \Omega_{0} \approx \gamma B_{0}^{\prime},
$$

yielding the solution $A_{1}=\exp \left(-i \Omega_{0} t / 2\right)$. This result, after substitution into Eq. (B2), yields

$$
i \frac{d A_{2}}{d t} \approx-\left(\frac{\Omega_{0}}{2}+i \lambda \Omega_{0}\right) A_{2}+\frac{\gamma}{2}\left(\delta B_{x}+i \delta B_{y}\right) e^{-i \Omega_{0} t / 2}
$$

or, by introducing $\psi=\left(2 a_{1}\right)^{1 / 2} A_{2} \exp \left(-i \Omega_{0} t / 2\right)$ :

$$
\frac{d \psi}{d t} \approx-\lambda \Omega_{0} \psi-\frac{\gamma\left(2 a_{1}\right)^{1 / 2}}{2}\left(i \delta B_{x}-\delta B_{y}\right) e^{-i \Omega_{0} t} .
$$

Finally, we separate the real and imaginary parts $(\psi=a+i b)$ in the last equation to get

$$
\begin{aligned}
& \frac{d a}{d t}=-\lambda \Omega_{0} a+\delta f^{a}(t), \\
& \frac{d b}{d t}=-\lambda \Omega_{0} b+\delta f^{b}(t),
\end{aligned}
$$

where

$$
\begin{array}{r}
\delta f^{a}(t)=\frac{\gamma\left(2 a_{1}\right)^{1 / 2}}{2}\left[\delta B_{y} \cos \left(\Omega_{0} t\right)-\delta B_{x} \sin \left(\Omega_{0} t\right)\right], \\
\delta f^{b}(t)=-\frac{\gamma\left(2 a_{1}\right)^{1 / 2}}{2}\left[\delta B_{x} \cos \left(\Omega_{0} t\right)+\delta B_{y} \sin \left(\Omega_{0} t\right)\right],
\end{array}
$$

and

$$
\left\langle\delta f^{a, b}(t) \delta f^{a, b}\left(t^{\prime}\right)\right\rangle=\frac{\gamma^{2} \eta a_{1}}{2} \delta\left(t-t^{\prime}\right) .
$$

The solution of the Langevin equations (B7) and (B8), together with ensemble averaging, yields

$$
\left\langle a^{2}\right\rangle=\left\langle b^{2}\right\rangle=\frac{\gamma^{2} \eta a_{1}}{4 \lambda \Omega_{0}}
$$

and, thus, using the definitions $\eta=\frac{2 k_{B} T \lambda}{\gamma \mu_{s}}$ and $\Omega_{0}=\gamma B_{0}^{\prime}$, we find

$$
2 \sigma^{2}=\left\langle|\psi|^{2}\right\rangle=\left\langle a^{2}\right\rangle+\left\langle b^{2}\right\rangle=\frac{k_{B} T a_{1}}{\mu_{s} B_{0}^{\prime}} .
$$

[1] J. Frenkel and J. Dorfman, Nature (London) 126, 274 (1930).

[2] F. M. Koehler et al., Chem. Commun. 32, 4862 (2009).

[3] I. Rabias et al., Biomicrofluidics 4, 024111 (2010).

[4] K. E. Scarberry, E. B. Dickerson, J. F. McDonald, and Z. J. Zhang, J. Am. Chem. Soc. 130, 10258 (2008).

[5] N. Parera Pera et al., Org. Biomol. Chem. 8, 2425 (2010).

[6] B. Hillebrands and J. Fassbender, Nature (London) 418, 493 (2002).

[7] C. H. Back et al., Science 285, 864 (1999).

[8] Th. Gerrits, H. A. M. van den Berg, J. Hohlfeld, L. Bär, and Th. Rasing, Nature (London) 418, 509 (2002).

[9] H. W. Schumacher, C. Chappert, P. Crozat, R. C. Sousa, P. P. Freitas, J. Miltat, J. Fassbender, and B. Hillebrands, Phys. Rev. Lett. 90, 017201 (2003).

[10] T. Seki, K. Utsumiya, Y. Nozaki, H. Imamura, and K. Takanashi, Nature Commun. 4, 1726 (2013).

[11] S. Sun, C. B. Murray, D. Weller, L. Folks, and A. Moser, Science 287, 1989 (2000).

[12] C.-R. Chang and J.-S. Yang, Phys. Rev. B 54, 11957 (1996).

[13] J. Miltat, G. Albuquerque, and A. Thiaville, in Spin Dynamics in Confined Magnetic Structures I, edited by B. Hillebrands and K. Ounadjela, Topics in Applied Physics Vol. 83 (Springer, Heidelberg, 2002), pp. 1-31.

[14] C. Thirion, W. Wernsdorfer, and D. Mailly, Nat. Mater. 2, 524 (2003).

[15] N. Barros, M. Rassam, H. Jirari, and H. Kachkachi, Phys. Rev. B 83, 144418 (2011).
[16] G. Klughertz, P.-A. Hervieux, and G. Manfredi, J. Phys. D: Appl Phys. 47, 345004 (2014).

[17] L. Friedland, Scholarpedia 4, 5473 (2009).

[18] B. Meerson and L. Friedland, Phys. Rev. A 41, 5233 (1990).

[19] W. K. Liu, B. Wu, and J. M. Yuan, Phys. Rev. Lett. 75, 1292 (1995).

[20] J. Fajans, E. Gilson, and L. Friedland, Phys. Rev. Lett. 82, 4444 (1999).

[21] J. Fajans, E. Gilson, and L. Friedland, Phys. Plasmas 6, 4497 (1999).

[22] L. Friedland, Phys. Rev. E 59, 4106 (1999).

[23] G. Manfredi and P.-A. Hervieux, Appl. Phys. Lett. 91, 061108 (2007).

[24] L. Cai, D. A. Garanin, and E. M. Chudnovsky, Phys. Rev. B 87, 024418 (2013).

[25] K. Rivkin and J. B. Ketterson, Appl. Phys. Lett. 89, 252507 (2006).

[26] Z. Wang and M. Wu, J. Appl. Phys. 105, 093903 (2009).

[27] R. Feynman, F. L. Vernon, and R. W. Hellwarth, J. Appl. Phys. 28, 49 (1957).

[28] M. Jamet, W. Wernsdorfer, C. Thirion, D. Mailly, V. Dupuis, P. Mélinon, and A. Pérez, Phys. Rev. Lett. 86, 4676 (2001).

[29] L. Friedland, Phys. Fluids B 4, 3199 (1992).

[30] A. Barak, Y. Lamhot, L. Friedland, and M. Segev, Phys. Rev. Lett. 103, 123901 (2009).

[31] O. Naaman, J. Aumentado, L. Friedland, J. S. Wurtele, and I. Siddiqi, Phys. Rev. Lett. 101, 117005 (2008). 
[32] E. Grosfeld and L. Friedland, Phys. Rev. E 65, 046230 (2002).

[33] I. Barth, L. Friedland, O. Gat, and A. G. Shagalov, Phys. Rev. A 84, 013837 (2011).

[34] L. D. Landau and E. M. Lifshitz, Quantum Mechanics (Nonrelativistic Theory), 3rd ed. (Butterworth-Heinemann, Oxford, 1977).

[35] J. Fajans, E. Gilson, and L. Friedland, Phys. Plasmas 8, 423 (2001).
[36] L. Néel, Ann. Géophys. 5, 99 (1949).

[37] W. F. Brown, Phys. Rev. 130, 1677 (1963).

[38] We also assume that the temperature is sufficiently small, so that the magnetic moments do not switch back and forth only because of the thermal agitation.

[39] I. Barth, L. Friedland, E. Sarid, and A. G. Shagalov, Phys. Rev. Lett. 103, 155001 (2009).

[40] H. Kesserwan, G. Manfredi, J.-Y. Bigot, and P.-A. Hervieux, Phys. Rev. B 84, 172407 (2011). 